\title{
Higher-rank tensor non-Abelian field theory: Higher-moment or subdimensional polynomial global symmetry, algebraic variety, Noether's theorem, and gauging
}

\author{
Juven Wang $\odot,{ }^{1, *} \mathrm{Kai} \mathrm{Xu}^{2, \dagger}$ and Shing-Tung Yau ${ }^{1,2,3, \sharp}$ \\ ${ }^{1}$ Center of Mathematical Sciences and Applications, Harvard University, Cambridge, Massachusetts 02138, USA \\ ${ }^{2}$ Department of Mathematics, Harvard University, Cambridge, Massachusetts 02138, USA \\ ${ }^{3}$ Department of Physics, Harvard University, Cambridge, Massachusetts 02138, USA
}

(Received 8 November 2019; accepted 12 February 2021; published 25 February 2021)

\begin{abstract}
With a view toward a fracton theory in condensed matter, we introduce a higher-moment polynomial degree-p global symmetry, acting on complex scalar/vector/tensor fields (e.g., ordinary or vector global symmetry for $\mathrm{p}=0$ and $\mathrm{p}=1$ respectively). We relate this higher-moment global symmetry of $n$-dimensional space, to a lower degree [either ordinary or higher-moment, e.g., degree-(p- $\ell$ )] subdimensional or subsystem global symmetry on layers of $(n-\ell)$-submanifolds. These submanifolds are algebraic affine varieties (i.e., solutions of polynomials). The structure of layers of submanifolds as subvarieties can be studied via mathematical tools of embedding, foliation, and algebraic geometry. We also generalize Noether's theorem for this higher-moment polynomial global symmetry. We can promote the higher-moment global symmetry to a local symmetry and derive a new family of higher-rank-m symmetric tensor gauge theory by gauging, with $m=p+1$. By further gauging a discrete $\mathbb{Z}_{2}^{C}$ charge conjugation (particle-hole) symmetry, we derive a general class of rank-m tensor non-Abelian gauge field theory (the gauge structure is noncommutative thus non-Abelian but not an ordinary group): a hybrid class of (symmetric or nonsymmetric) higher-rank-m tensor gauge theory and antisymmetric tensor topological field theory, generalizing Wang and Xu [Ann. Phys. 424, 168370 (2021)], interplaying between gapless and gapped sectors.
\end{abstract}

DOI: 10.1103/PhysRevResearch.3.013185

\section{INTRODUCTION}

Fracton orders [1,2] are the new kinds of orders in manybody quantum matter systems. Fracton orders are defined physically by exhibiting some of (if not all of) the following properties: ${ }^{1}$

(1) For gapped fractons, their ground state degeneracy (GSD) is similar to topological order [5] with GSD depending on the topology of base space or spatial manifolds. Moreover, they have extensive GSD depending on the system size and the details of lattice sites and cutoffs $[6,7]$.

(2) Fracton orders can also have excitations, either being immobile in isolation or being mobile moving along in lower dimensions or subdimensions $[8,9]$.

\footnotetext{
*Corresponding author: jw@cmsa.fas.harvard.edu

†kaixu@math.harvard.edu

‡yau@math.harvard.edu
}

Published by the American Physical Society under the terms of the Creative Commons Attribution 4.0 International license. Further distribution of this work must maintain attribution to the author(s) and the published article's title, journal citation, and DOI.

${ }^{1}$ We focus on the limited references essential to the construction of our theories. We apologize for potentially leaving out some other important works from the References. For more references in the condensed matter literature, readers can find them in the reviews $[1,2]$. For a short historical account of gauge theory and earlier references, starting from the basics of Maxwell electromagnetism and the Weyl gauge principle [3], readers can find these in Ref. [4].
(3) Fracton orders are associated with the long-range entangled phases of quantum matter, obtainable by dynamically gauging the subsystem global symmetries or subdimensional global symmetries of the full quantum systems [9-11] (see also earlier work $[12,13]$ before the fracton concept is introduced).

Motivated by the fracton order in condensed matter, recently two of the present authors introduced a hybrid family of tensor gauge field theories [4] mixing between the antisymmetric tensor gauge fields and symmetric higher-rank tensor gauge fields in a delicate way. Their purpose was to formulate the first toy model of a gauge theory with a non-Abelian continuous gauge structure for fracton order in condensed matter (see reviews $[1,2]$ ). The toy model [4] suggested an interplay between the following:

(1) The gapped antisymmetric tensor gauge topological quantum field theory (TQFT) with topological order and

(2) The gapless symmetric higher-rank tensor gauge theory with gapless higher-spin U(1)-gauge photon-like modes.

The higher-rank tensor gauge theory in Ref. [4] combines the features of the following:

(1) Antisymmetric tensor topological field theory (TQFT): We adopt a continuum TQFT formulation of groupcohomology topological gauge theory (known as DijkgraafWitten theory or twisted gauge theory [14]) by antisymmetric tensor differential form gauge fields (i.e., Kalb-Ramond fields [15]). In Ref. [4] and here, we mainly use a particular continuum TQFT formalism setup and notations presented in Refs. [16-19] that can capture all finite Abelian unitary gauge group and some non-Abelian unitary gauge group of 
Dijkgraaf-Witten theory with the group-cohomology cocycle twist. (See also other related general formulations for nondynamical gauge background theories $[20,21]$ and references therein.)

(2) Symmetric tensor field theory: We will apply only a specific class of symmetric higher-rank tensor gauge theories or higher-spin theories studied in the condensed matter literature, e.g., Refs. [22-27], largely inspired by Pretko's work.

Reference [4] finds a class of symmetric higher-rank tensor gauge field theory (from Model 2 of the symmetric tensor field theory) by gauging a higher-moment Abelian vector global symmetry $\mathrm{U}(1)_{x_{(n)}}^{2}$ and an ordinary 0 -form global symmetry known as a $\mathbb{Z}_{2}^{C}$-charge conjugation (particle-hole) symmetry. The higher-moment symmetry $\mathrm{U}(1)_{x_{(n)}}$ and the $\mathbb{Z}_{2}^{C}$-charge conjugation symmetry do not commute. As we shall elaborate below, they form a semidirect product (denoted $\ltimes$ ) structure. Reference [4] dynamically gauges the $\mathbb{Z}_{2}^{C}$-charge conjugation symmetry to gain a non-Abelian gauge structure: ${ }^{3}$

$$
\left[\mathbb{Z}_{2}^{C} \ltimes\left(\mathrm{U}(1)_{x_{(n)}}\right)\right] .
$$

This gauge structure is the first example in the fracton order literature satisfying the properties below: ${ }^{4}$

(1) Compact

(2) Continuous 5

\footnotetext{
${ }^{2}$ Here we denote a vector global symmetry along $n$ dimensions as $\mathrm{U}(1)_{x_{(n)}}$. The $\mathrm{U}(1)_{x_{(d+1)}}$ means the vector global symmetry in a $d+1$-dimensional spacetime, and the $\mathrm{U}(1)_{x_{(d)}}$ means the vector global symmetry in a $d$-dimensional space. The $x$ means being set of Cartesian coordinates for the spacetime. Throughout this article, we focus on only a flat Euclidean or Minkowski spacetime $\left(\mathbb{R}^{d+1}\right.$ or $\mathbb{R}^{1, d}$ ) with Cartesian coordinates. The Cartesian coordinates can be easily realized in square, rectangular, or cubic lattices in condensed matter systems. On the other hand, it is difficult to imagine how to rewrite the higher-moment global symmetry in curved coordinates such as spherical or cylindrical coordinates, and how to realize them in a lattice system with energy cutoffs in condensed matter. The $d+1 \mathrm{~d}$ means the $d+1$ spacetime dimensions, with $d$ spatial and one time dimensions. The $D \mathrm{D}$ means the $D$ spacetime dimensions. The $\bar{D} \mathrm{D}$ means the $\bar{D}$ space dimensions. We denote by $d+1 \mathrm{D}$ the $d$ spatial and one time dimensions.

${ }^{3}$ This $\mathbb{Z}_{2}^{C} \ltimes\left(\mathrm{U}(1)_{x_{(n)}}\right)$ is not quite an ordinary group structure. However, because the polynomial symmetry operation $\mathrm{U}(1)_{x_{(n)}}$ does not commute with the unitary $\mathbb{Z}_{2}^{C}$ symmetry, we still stick to the standard convention to call the noncommutative structure a non-Abelian structure. We also call the noncommutative gauge structure (although not a gauge group) a non-Abelian gauge structure. We will later dynamically gauge the $\mathbb{Z}_{2}^{C}$ and the polynomial global symmetry $\mathrm{U}(1)_{x_{(n)}}$ all together. We emphasize that the discrete charge conjugation $\mathbb{Z}_{2}^{C}$ is a unitary global symmetry. This $\mathbb{Z}_{2}^{C}$ is distinct from another discrete symmetry: antiunitary time-reversal symmetry (e.g., $\mathbb{Z}_{2}^{T}$ ).

${ }^{4}$ We denote the global symmetry in brackets [...] to imply that it is dynamically gauged.

${ }^{5}$ We remind the readers that there are alternative means to construct non-Abelian fracton orders with discrete gauge structures on a lattice [28-32] (e.g., discrete gauge theories) instead of continuous gauge structures. It is possible to Higgs down our model with continuous gauge structures to obtain higher-rank non-Abelian tensor field theories with non-Abelian discrete gauge structures [33].
}

(3) Non-Abelian (gauging $\mathbb{Z}_{2}^{C}$ ) or Abelian (not gauging $\left.\mathbb{Z}_{2}^{C}\right)$, with two disconnected pieces in the gauge structure due to $\mathbb{Z}_{2}^{C}$.

The $\mathrm{U}(1)_{x_{(n)}}$ actually means there are $n$-independent vector directions $x_{i}$ with $i=1, \ldots, n$. It is easier to understand $\mathrm{U}(1)_{x_{(n)}}$ before gauging it. Thus, let us first recover the gauge structure Eq. (1.1) from the ungauged global symmetry:

$$
\mathbb{Z}_{2}^{C} \ltimes\left(\mathrm{U}(1) \times \mathrm{U}(1)_{x_{(n)}}\right) .
$$

We can perform an ordinary 0 -form global symmetry $\mathrm{U}(1)$ and a vector global symmetry $\mathrm{U}(1)_{x_{(n)}}$ transformation by transforming a complex matter field $\Phi \in \mathbb{C}$ (following the pioneering work of Pretko $[4,27])$

$$
\Phi \rightarrow e^{i Q(x)} \Phi=e^{i\left(\Lambda_{i} x_{i}+\Lambda_{0}\right)} \Phi .
$$

(1) When the $\Lambda_{i}$ (as a constant, independent of spacetime coordinates) is nonzero, we have a degree-1 polynomial $Q(x)=\left(\Lambda_{i} x_{i}+\Lambda_{0}\right)$ of $x$ which $\Lambda_{i} x_{i}$ specifies the $\mathrm{U}(1)_{x_{(n)}}$ vector global symmetry, while $\Lambda_{0}$ specifies the U(1) ordinary global symmetry. Since there are $n$ independent $\Lambda_{i} x_{i}$, we have indeed several copies of commuting $\mathrm{U}(1)_{x_{j}}$-vector global symmetry:

$$
\begin{aligned}
\mathrm{U}(1)_{x_{(n)}}:= & \mathrm{U}(1)_{x_{1}} \times \mathrm{U}(1)_{x_{2}} \\
& \times \cdots \times \mathrm{U}(1)_{x_{n}}=\prod_{j=1}^{n} \mathrm{U}(1)_{x_{j}} .
\end{aligned}
$$

We name this global symmetry a degree- 1 global symmetry, due to the degree-1 polynomial $Q(x)=\left(\Lambda_{i} x_{i}+\Lambda_{0}\right)$.

(2) When the $\Lambda_{i}$ is zero, we have a degree- 0 polynomial $\left(\Lambda_{0}\right)$ independent of $x$. We name this global symmetry a degree-0 global symmetry due to the degree-0 polynomial $Q(x)=\Lambda_{0}$.

If we gauge only the vector global symmetry $\mathrm{U}(1)_{x_{(n)}}$ but not the ordinary symmetry $\mathrm{U}(1)$, we gain the $\left[\mathrm{U}(1)_{x_{(n)}}\right]$ gauge structure, while the remained $\mathrm{U}(1)$ is neither gauged nor global symmetry anymore. This particular way of gauging $\left[\mathrm{U}(1)_{x_{(n)}}\right]$ introduces the compact symmetric rank-2 tensor gauge field $A_{i j}$. Reference [4] dynamically gauges the discrete $\mathbb{Z}_{2}^{C}$ symmetry which flips

$$
A_{i j} \rightarrow-A_{i j}
$$

to gain the non-Abelian gauge structure.

To help the readers understanding the non-Abelian gauge structure $\left[\mathbb{Z}_{2}^{C} \ltimes\left(\mathrm{U}(1)_{x_{(n)}}\right)\right]$ in Eq. (1.1), here we show the noncommutative symmetry operations between the $\mathrm{U}(1)_{x_{(n)}}$ vector global symmetry transformation (Fig. 1) and the $\mathbb{Z}_{2}^{C}$ charge conjugation (particle-hole) symmetry transformation on a complex bosonic scalar field $\Phi(x) \in \mathbb{C}$ in cartoon figures; see Fig. 2.

In this work, we follow the setup in Ref. [4] and proceed to develop other families of theories. We consider the following generalization:

(1) Higher-moment global symmetry for a complex scalar charge field $\Phi \in \mathbb{C}$ : A general polynomial degree- $(m-1)$ 

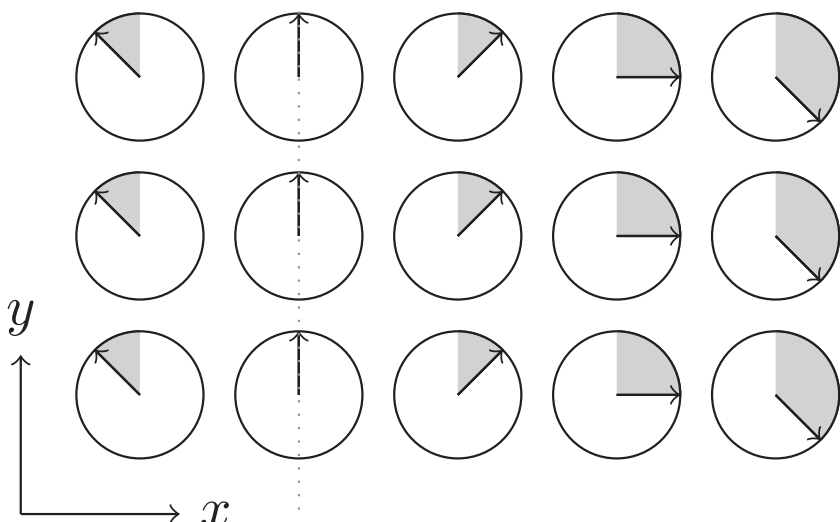

$$
x=0
$$

FIG. 1. The vector global symmetry Eq. (1.3) belongs to a generalized class of higher-moment symmetry Eq. (1.5). For demonstration, here we show various $\Phi(x)$ fields sitting on discretized lattice points on the $(x, y)$ plane. The vector $\mathrm{U}(1)$ global symmetry transformation acts on the complex charged matter $\Phi(x) \in \mathbb{C}$ (the rotor fields) as Eq. (1.3): $\Phi \rightarrow e^{i Q(x)} \Phi:=e^{i \Lambda \cdot x} \Phi$. The angle $\Lambda \cdot x$ depends on a reference point (say, $x=0$ ) and the distance $x$ away from the reference point. The clockwise angle (drawn in the gray area) away from the 12 o'clock direction implies the complex phase of $\Phi(x) \in \mathbb{C}$.

global symmetry ${ }^{6}$ on $\Phi$ allows a symmetry transformation

$$
\Phi \rightarrow e^{i Q(x)} \Phi:=e^{i\left(\Lambda_{i_{1}, \ldots, i_{\mathrm{m}-1}} x_{i_{1}} \ldots x_{i_{\mathrm{m}-1}}+\cdots+\Lambda_{i, j} x_{i} x_{j}+\Lambda_{i} x_{i}+\Lambda_{0}\right)} \Phi .
$$

Here all $\Lambda_{\ldots}$ in Eq. (1.5) are constants independent of spacetime coordinates. When we gauge such a higher-moment polynomial degree- $(\mathrm{m}-1)$ global symmetry, we will introduce a rank-m compact symmetric tensor gauge field $A_{i_{1}, \ldots, i_{\mathrm{m}}}$. This result is done in Sec. II.

Let us generalize the notation of the symmetry in Eq. (1.4) into

$$
\mathrm{U}(1)_{x_{\left(\begin{array}{c}
n \\
M
\end{array}\right)}^{M}}:=\prod_{\left\{j_{1}, \ldots, j_{M}\right\}} \mathrm{U}(1)_{x_{j_{1}}, \ldots, x_{j_{M}}},
$$

for each of different $\Lambda_{j_{1}, \ldots, j_{M}}$ independent coefficients of the symmetry generators from a degree $M$ polynomial. Therefore, for the full Eq. (1.5), if every coefficient $\Lambda$ is allowed, then by combining all the symmetry generators from degree $0,1, \ldots$ to degree $m-1$, we have the full symmetry structure:

$$
\mathrm{U}(1)_{x_{\left(\begin{array}{c}
n \\
\mathrm{~m}-1
\end{array}\right)}^{\mathrm{m}-1}} \times \cdots \times \mathrm{U}(1)_{x_{(n)}} \times \mathrm{U}(1)=\prod_{M=0}^{\mathrm{m}-1} \mathrm{U}(1)_{x_{\left(\begin{array}{c}
n \\
M
\end{array}\right)}^{M}} .
$$

(2) Higher-moment global symmetry for a complex vector charge field $\Phi_{I} \in \mathbb{C}$ (where the vector index is $I$, but each $\Phi_{I}$ still is a complex scalar field): A general polynomial

\footnotetext{
${ }^{6}$ Throughout our work, we define $(\mathrm{m}-1) \equiv \mathrm{p}$ for some integers $\mathrm{m} \geqslant 1$ and $\mathrm{p} \geqslant 0$. The reason to choose $\mathrm{m} \geqslant 1$ will become clear later when we require constructing the Lagrangian from the mth derivative term $\partial^{\mathrm{m}} \log \Phi$.
}

degree- $(m-1)$ global symmetry on $\Phi_{I}$ allows a symmetry transformation

$$
\begin{aligned}
\Phi_{I} & \rightarrow e^{i Q_{I}(x)} \Phi_{I} \\
& :=e^{i\left(\Lambda_{I ; i_{1}, \ldots, i_{\mathrm{m}-1}} x_{i_{1}} \ldots x_{i_{\mathrm{m}}-1}+\cdots+\Lambda_{I ; i} x_{i} x_{j}+\Lambda_{I ; i} x_{i}+\Lambda_{I ; 0}\right)} \Phi_{I} .
\end{aligned}
$$

The degree- $(\mathrm{m}-1) \quad$ polynomial $\quad Q_{I}(x)=$ $\left(\Lambda_{I ; i_{1}, \ldots, i_{\mathrm{m}}-1} x_{i_{1}} \ldots x_{i_{\mathrm{m}}-1}+\cdots+\Lambda_{I ; i, j} x_{i} x_{j}+\Lambda_{I ; i} x_{i}+\Lambda_{I ; 0}\right)$ has an index $I$.

(3) Higher-moment global symmetry for a complex rank$M$ tensor charge field $\Phi_{I_{1}, \ldots, I_{M}} \in \mathbb{C}$ (where the tensor index is $I_{1}, \ldots, I_{M}$, but each $\Phi_{I_{1}, \ldots, I_{M}}$ still is a complex scalar field): A general polynomial degree- $(\mathrm{m}-1)$ global symmetry on $\Phi_{I_{1}, \ldots, I_{M}}$ allows a symmetry transformation

$$
\Phi_{I_{1}, \ldots, I_{M}} \rightarrow e^{i Q_{I_{1}, \ldots, I_{M}}(x)} \Phi_{I_{1}, \ldots, I_{M}} .
$$

(4) For all the above theories of higher-moment global symmetries, we construct their corresponding gauge theories by dynamically gauging the global symmetry (see Fig. 3). We introduce the Abelian and non-Abelian tensor gauge field and their gauge-invariant or covariant field strength tensor. We can use the field strength to construct the gauge-invariant kinetic Lagrangian term of the non-Abelian tensor gauge theory, shown in Secs. II A 2 and II A 3.

We also notice that Refs. [34,35] had also attempted to study the polynomial types or higher-moment types of global symmetries systematically. However, our motivations are somehow different from Ref. [34], and our framework is somehow different from Ref. [35]. We do not yet know a precise correspondence between our results $[4,33,36]$ and theirs [34,35].

Our theory can be formulated as compatible with or without Euclidean, Poincaré, isotropic, or anisotropic symmetry in the $d+1-\mathrm{D}$ spacetime, at least in ultraviolet high or intermediate energy field theory, but not yet to a lattice cutoff scale; see more discussions in various versions of theories in Ref. [4]. Thus for Euclidean or Poincaré symmetry, we need to choose the $n$ dimensions in $\mathrm{U}(1)_{x_{(n)}}$ as $n=d+1$ for dimensions. For an anisotropic symmetry, we can choose the $n$ dimensions in $\mathrm{U}(1)_{x_{(n)}}$ as $n \leqslant d$ for dimensions. Below we shall keep the general index $n$ in $\mathrm{U}(1)_{x_{(n)}}$, and leave the substitution of $n$ free (to $n=d+1$ or $n \leqslant d$ ) based on the specific needs of readers.

In a companion work, we explore the types of sigma model that can interpolate between the disorder phases (as the present higher-rank tensor non-Abelian gauge theories) and the ordered phases. Similar to the famous quantum phase transition between insulator [U(1) symmetry disorder described by a topological gauge theory or a disordered Sigma model] and superfluid/superconductivity [U(1) global/gauge symmetry-breaking order described by a Sigma model with a U(1) target space with Goldstone modes], we can explore phase structures of order-disorder phases by developing a Sigma model [36]. A recent work studies the superfluid phase of a pure Abelian fractonic matte field theory without gauge fields [37], while we study instead both the ordered phase (superfluid and a Sigma model) and the disordered phase of non-Abelian gauged fractonic matte field theories with gauge fields [36]. 


\section{SCALAR CHARGE, HIGHER-MOMENT POLYNOMIAL DEGREE-(M-1) GLOBAL SYMMETRY, AND RANK-M GAUGE THEORY}

First, we consider how to gauge the following global symmetry for s scalar field $\Phi$ on the $\mathbb{R}^{n}$, the $n$-dimensional space or spacetime:

$$
\Phi \rightarrow e^{i Q(x)} \Phi,
$$

where $Q(x)$ is a polynomial with degree at most $(\mathrm{m}-1) \equiv \mathrm{p}$ for some integers $m \geqslant 1$ and $p \geqslant 0$, say,

$$
Q(x):=\left(\Lambda_{i_{1}, \ldots, i_{\mathrm{m}-1}} x_{i_{1}} \ldots x_{i_{\mathrm{m}-1}}+\cdots+\Lambda_{i, j} x_{i} x_{j}+\Lambda_{i} x_{i}+\Lambda_{0}\right) .
$$

Note that the gauge transformation can be written as

$$
\log \Phi \rightarrow \log \Phi+i Q(x),
$$

and the only invariant quantity under this transformation is

$$
\partial^{\mathrm{m}} \log \Phi,
$$

which is an order $\mathrm{m}$ symmetric tensor whose components are

$$
\partial_{i_{1}} \cdots \partial_{i_{\mathrm{m}}} \log \Phi
$$

where the spacetime indices $i_{k} \in\{1,2, \ldots, n\}$, with $k \in$ $\{1,2, \ldots, m\}$. In the next subsection, before gauging this higher-moment symmetry, we construct the covariant operator $\left[P_{i_{1}, \ldots, i_{m}}\right.$ in Eq. (2.7)]. After gauging this higher-moment symmetry, we also construct the gauge-invariant operator (such as the Abelian gauge field strength in Sec. II A 2) or gauge covariant operator [such as the covariant derivative on
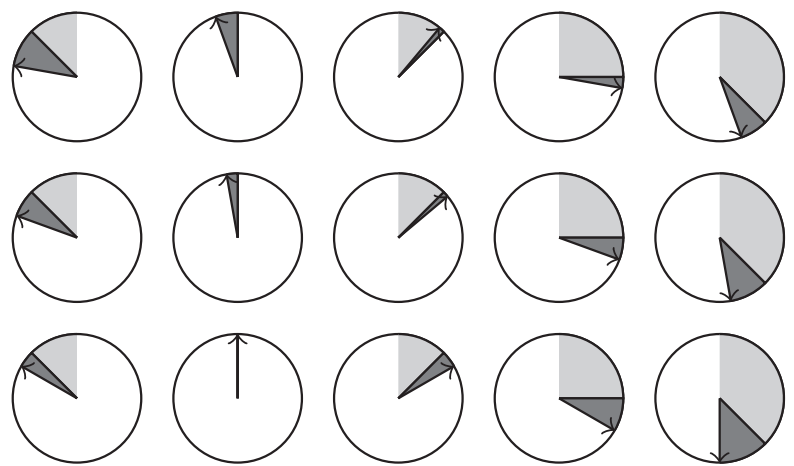

FIG. 3. A demonstration of the local fluctuations (the dark gray) that deviate away from the vector (degree-1 polynomial) global symmetry of Eq. (1.3) or higher-moment polynomial global symmetry of Eq. (1.5). The light gray indicates higher-moment degree-(m-1) global symmetry transformations (here we show $\mathrm{m}=1$ ). We will introduce a gauge theory to compensate the local gauge fluctuation in Sec. II by introducing a rank-m tensor gauge field $A_{i_{1}, \ldots, i_{\mathrm{m}}}$ [Eq. (2.16)] mediating between the matter $\Phi$ fields.

the matter field $D_{i_{1}, \ldots, i_{m}}[\{\Phi\}]$ in Eq. (2.12) or the non-Abelian gauge field strength in Sec. II A 3].

\section{A. Polynomial with arbitrary degree}

By the law of differentiation, we know that

$$
\partial_{i_{1}} \cdots \partial_{i_{\mathrm{m}}} \log \Phi=\frac{P_{i_{1}, \ldots, i_{\mathrm{m}}}\left(\Phi, \ldots, \partial^{\mathrm{m}} \Phi\right)}{\Phi^{\mathrm{m}}},
$$

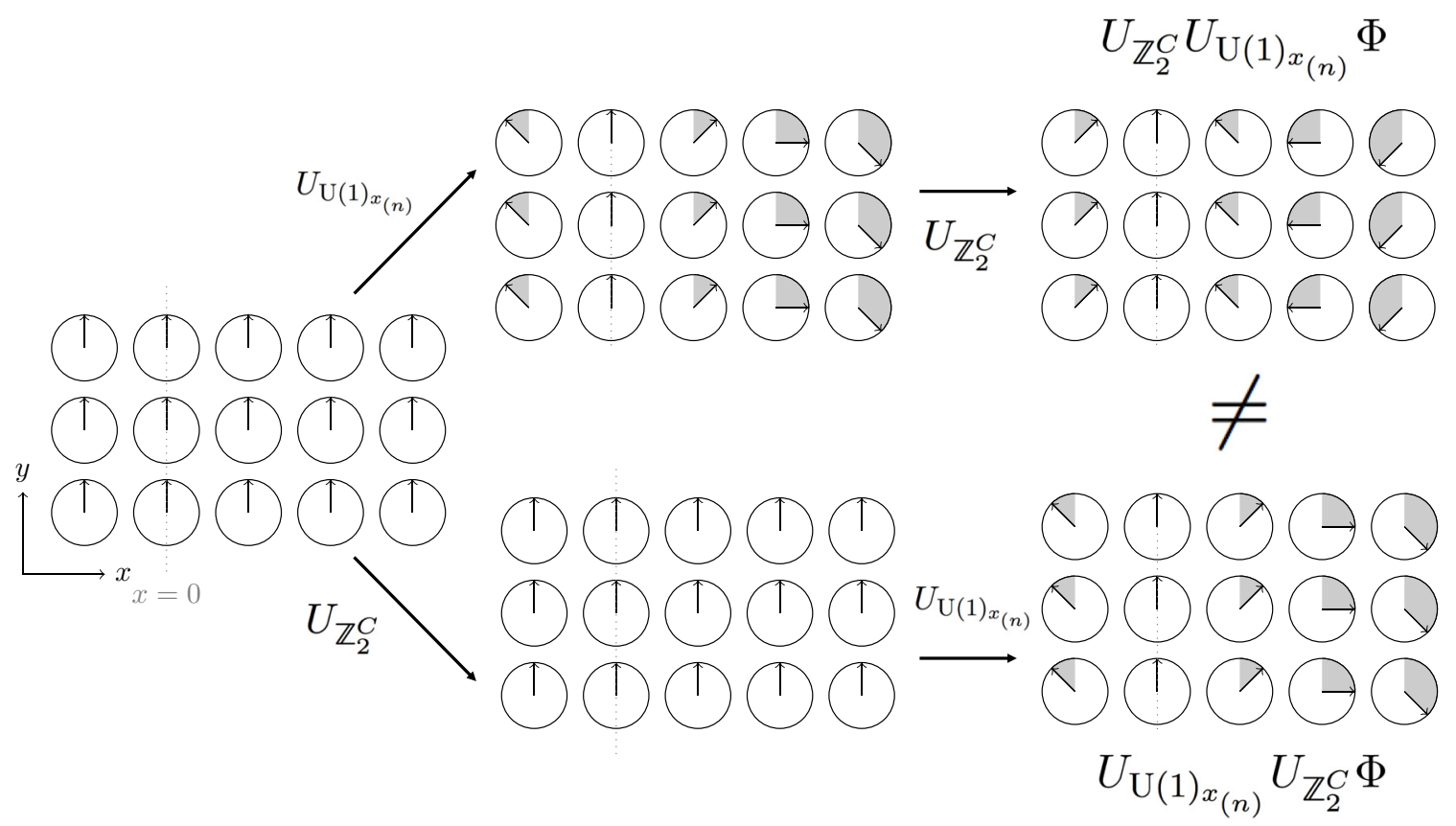

FIG. 2. Following the setup of Fig. 1, here we show the noncommutative nature of the $\mathbb{Z}_{2}^{C} \ltimes \mathrm{U}(1)_{x_{(n)}}$ global symmetry by performing the $U_{\mathbb{Z}_{2}^{C}} U_{\mathrm{U}(1)_{x(n)}} \Phi$ transformation on the top route, versus the $U_{\mathrm{U}(1)_{x(n)}} U_{\mathbb{Z}_{2}^{C}} \Phi$ transformation on the bottom route. The top route shows $U_{\mathbb{Z}_{2}^{c}} U_{\mathrm{U}(1)_{x_{(n)}}} \Phi=U_{\mathbb{Z}_{2}^{C}}\left(e^{i Q(x)} \Phi\right)=e^{i Q(x)} \Phi^{\dagger}$. The bottom route shows $U_{\mathrm{U}(1)_{x_{(n)}}} U_{\mathbb{Z}_{2}^{c}} \Phi=U_{\mathrm{U}(1)_{x_{(n)}}}\left(\Phi^{\dagger}\right)=e^{-i Q(x)} \Phi^{\dagger}$. In summary, we demonstrate $U_{\mathbb{Z}_{2}^{C}} U_{\mathrm{U}(1)_{x}(n)} \Phi \neq U_{\mathrm{U}(1)_{x_{(n)}}} U_{\mathbb{Z}_{2}^{C}} \Phi$. Indeed, the noncommutative nature between the $\mathbb{Z}_{2}^{C}$ and the higher-moment global symmetry still holds, even for a more general polynomial global symmetry [such as Eq. (1.5)'s transformation $\Phi \rightarrow e^{i Q(x)} \Phi:=e^{i\left(\Lambda_{i_{1}, \ldots, i_{\mathrm{m}-1} x_{i_{1}} \ldots x_{i} \mathrm{~m}-1}+\cdots+\Lambda_{i, j} x_{i} x_{j}+\Lambda_{i} x_{i}+\Lambda_{0}\right)} \Phi$ ]. 
so this order $\mathrm{m}$ tensor $P$ transforms as

$$
P_{i_{1}, \ldots, i_{\mathrm{m}}} \rightarrow e^{i \mathrm{~m} Q(x)} P_{i_{1}, \ldots, i_{\mathrm{m}}} .
$$

Under a more general gauge transformation

$$
\Phi \rightarrow e^{i \eta(x)} \Phi,
$$

we find that

$$
\log \Phi \rightarrow \log \Phi+i \eta(x) .
$$

We shorthand $\partial_{i_{1}} \cdots \partial_{i_{\mathrm{m}}}:=\partial^{\mathrm{m}}$ so

$$
\partial^{\mathrm{m}} \log \Phi \rightarrow \partial^{\mathrm{m}} \log \Phi+i \partial^{\mathrm{m}} \eta(x) .
$$

This implies

$$
\begin{aligned}
P_{i_{1}, \ldots, i_{\mathrm{m}}}\left(\Phi, \ldots, \partial^{\mathrm{m}} \Phi\right) \rightarrow & e^{i \mathrm{~m} \eta(x)}\left[P_{i_{1}, \ldots, i_{\mathrm{m}}}\left(\Phi, \ldots, \partial^{\mathrm{m}} \Phi\right)\right. \\
& \left.+i \partial_{i_{1}} \cdots \partial_{i_{\mathrm{m}}} \eta(x) \Phi^{\mathrm{m}}\right] .
\end{aligned}
$$

Therefore it is natural to introduce the connection-like symmetric rank-m tensor gauge field $A_{i_{1}, \ldots, i_{\mathrm{m}}}$ and higher covariant derivative

$$
D_{i_{1}, \ldots, i_{\mathrm{m}}}[\{\Phi\}]:=P_{i_{1}, \ldots, i_{\mathrm{m}}}\left(\Phi, \ldots, \partial^{\mathrm{m}} \Phi\right)-i g A_{i_{1}, \ldots, i_{\mathrm{m}}} \Phi^{\mathrm{m}},
$$

where we implicitly sum over all possible indices as

$$
\begin{aligned}
\sum_{\left\{i_{1}, \ldots, i_{\mathrm{m}}\right\}} D_{i_{1}, \ldots, i_{\mathrm{m}}}[\{\Phi\}]:= & \sum_{\left\{i_{1}, \ldots, i_{\mathrm{m}}\right\}}\left[P_{i_{1}, \ldots, i_{\mathrm{m}}}\left(\Phi, \ldots, \partial^{\mathrm{m}} \Phi\right)\right. \\
& \left.-i g A_{i_{1}, \ldots, i_{\mathrm{m}}} \Phi^{\mathrm{m}}\right],
\end{aligned}
$$

which transforms covariantly under a general gauge transformation

$$
\Phi \rightarrow e^{i \eta(x)} \Phi .
$$

This implies that the tensor gauge field $A$ transforms as

$$
A \rightarrow A+\frac{1}{g} \partial^{\mathrm{m}} \eta \text {. }
$$

More precisely, components by components, it transforms as

$$
\begin{aligned}
A_{i_{1}, \ldots, i_{\mathrm{m}}} & \rightarrow A_{i_{1}, \ldots, i_{\mathrm{m}}}+\frac{1}{g} \partial_{i_{1}, i_{2}, \ldots, i_{\mathrm{m}-1}, i_{\mathrm{m}}} \eta \\
& :=A_{i_{1}, \ldots, i_{\mathrm{m}}}+\frac{1}{g} \partial_{i_{1}} \partial_{i_{2}} \cdots \partial_{i_{\mathrm{m}-1}} \partial_{i_{\mathrm{m}}} \eta .
\end{aligned}
$$

So a gauge-invariant term in the Lagrangian, involving the interactions between the gauge field and the scalar field, is

$$
\begin{aligned}
\left|D_{i_{1}, \ldots, i_{\mathrm{m}}}[\{\Phi\}]\right|^{2}:= & {\left[P_{i_{1}, \ldots, i_{\mathrm{m}}}\left(\Phi, \ldots, \partial^{\mathrm{m}} \Phi\right)-i g A_{i_{1}, \ldots, i_{\mathrm{m}}} \Phi^{\mathrm{m}}\right] } \\
& \times\left[P^{i_{1}, \ldots, i_{\mathrm{m}}}\left(\Phi^{\dagger}, \ldots, \partial^{\mathrm{m}} \Phi^{\dagger}\right)\right. \\
& \left.+i g A^{i_{1}, \ldots, i_{\mathrm{m}}}\left(\Phi^{\dagger}\right)^{\mathrm{m}}\right] .
\end{aligned}
$$

\section{Different meanings of "gauging"}

We should mention that our gauging procedure is a generalization of Ref. [27], but our gauging procedure may be different from some others in the literature $[9-11,38,39]$. This implies that the meaning of "gauging" actually is not the most unique refined statement-there can be different ways of "gauging" although the initial global symmetry is the same. "Gauging" can imply many different things:
(1) Promoting a global symmetry to a local symmetry, and the local symmetry fluctuation is absorbed by the gauge transformations of dynamical gauge fields. (This is our way of gauging the higher-moment global symmetry $[4,36]$.)

(2) Coupling the symmetry generator (the charge operator or the charge current) of the higher-moment symmetry to a background field. Then in the partition function, one makes the background field dynamical by summing over all the allowed background field configurations. This is also related to the orbifold procedure in field theory or string theory.

(3) Gauging may also be interpreted as the condensation of the "charged object" $\mathcal{O}$ of a global symmetry. The condensation means that the "charged object" becomes part of the property of the ground state wave function. Suppose the "charged object" $\mathcal{O}$ to be a local point operator or an extended (line, surface, etc.) operator, then the ground state $\left|\Psi_{\text {ground state }}\right\rangle$ (i.e., vacuum) with the condensed "charged object" $\mathcal{O}$ means that in the quantum mechanical sense, the ground state is in a coherent state

$$
\hat{\mathcal{O}}\left|\Psi_{\text {ground state }}\right\rangle \propto\left|\Psi_{\text {ground state }}\right\rangle .
$$

The $\hat{\mathcal{O}}$ is correspondingly a local or an extended quantum mechanical operator of the "charged object" $\mathcal{O}$ in field theory. Intuitively $\hat{\mathcal{O}}$ can be created and annihilated from the vacuum for free- $\hat{\mathcal{O}}$ can pop out or pop into the vacuum:

$$
\left\langle\Psi_{\text {ground state }}|\hat{\mathcal{O}}| \Psi_{\text {ground state }}\right\rangle \propto\langle\hat{\mathcal{O}}\rangle \neq 0,
$$

which is known as the condensation in the vacuum.

All these procedures are related to "gauging," and although we can gauge the same initial global symmetry, different gauging procedures may (or may not) give rise to different types of gauge theories. In our work, we study the gauging from the perspectives of continuum field theory. We do not yet attempt to make connections to other "gauging" procedures done on the lattice [9-11,39], but leave that for future work.

\section{Abelian gauge field strength and tensor gauge theory}

Following Sec. II A, to construct a rank- $(\mathrm{m}+1)$ gaugeinvariant Abelian gauge field strength, we simply define

$$
F_{\mu, v, i_{2}, \ldots, i_{\mathrm{m}}}:=\partial_{\mu} A_{v, i_{2}, \ldots, i_{\mathrm{m}}}-\partial_{\nu} A_{\mu, i_{2}, \ldots, i_{\mathrm{m}}} .
$$

Here $F_{\mu, v, i_{1}, \ldots, i_{\mathrm{m}}}$ is antisymmetric respect to $\mu \leftrightarrow \nu$. It is easy to check the gauge invariance of $F_{\mu, v, i_{1}, \ldots, i_{\mathrm{m}}}$ under the Abelian gauge transformation Eq. (2.16):

$$
\begin{aligned}
F_{\mu, v, i_{2}, \ldots, i_{\mathrm{m}} \rightarrow} & \partial_{\mu}\left(A_{\nu, i_{2}, \ldots, i_{\mathrm{m}}}+\frac{1}{g} \partial_{\nu} \partial_{i_{2}} \cdots \partial_{i_{\mathrm{m}}}\right) \\
& -\partial_{\nu}\left(A_{\mu, i_{2}, \ldots, i_{\mathrm{m}}}+\frac{1}{g} \partial_{\mu} \partial_{i_{2}} \cdots \partial_{i_{\mathrm{m}}} \eta\right) \\
= & F_{\mu, \nu, i_{2}, \ldots, i_{\mathrm{m}}} .
\end{aligned}
$$

It is easy to construct the gauge-invariant kinetic Lagrangian term

$$
\left|\hat{F}_{\mu, v, i_{2}, \ldots, i_{\mathrm{m}}}\right|^{2}:=F_{\mu, v, i_{2}, \ldots, i_{\mathrm{m}}} F^{\mu, v, i_{2}, \ldots, i_{\mathrm{m}}} .
$$

\section{Non-Abelian gauge field strength and tensor gauge theory}

We can also promote the Abelian gauge field strength Eq. (2.20) to a non-Abelian gauge field strength; we follow 
the trick of Ref. [4] by gauging the ordinary 0-form $\mathbb{Z}_{2}^{C}$-charge conjugation global symmetry. The $\mathbb{Z}_{2}^{C}$ acts on the rank-m tensor gauge field via

$$
A_{i_{1}, \ldots, i_{\mathrm{m}}} \rightarrow-A_{i_{1}, \ldots, i_{\mathrm{m}}} .
$$

By promoting the global $\mathbb{Z}_{2}^{C}$ to a local symmetry, we introduce a 1 -form $\mathbb{Z}_{2}^{C}$-gauge field $C$ coupling to the 0 -form symmetry $\mathbb{Z}_{2}^{C}$-charged object $A_{i_{1}, \ldots, i_{\mathrm{m}}}$ with a $g_{c}$ coupling. The $\mathbb{Z}_{2}^{C}$ local gauge transformation is

$$
A_{i_{1}, \ldots, i_{\mathrm{m}}} \rightarrow e^{i \gamma_{c}(x)} A_{i_{1}, \ldots, i_{\mathrm{m}}}, \quad C_{\nu} \rightarrow C_{\nu}+\frac{1}{g_{c}} \partial_{\nu} \gamma_{c}(x) .
$$

Note that $A_{i_{1}, \ldots, i_{\mathrm{m}}}$ is real-valued, so a generic $e^{i \gamma_{c}(x)}$ complexifies the $A_{i_{1}, \ldots, i_{\mathrm{m}}}$. However, what we can do is restrict gauge transformation so it is only $\mathbb{Z}_{2}^{C}$-gauged [not $\mathrm{U}(1)^{C}$-gauged],

$$
e^{i \gamma_{c}(x)}:=(-1)^{\gamma_{c}^{\prime}(x)} \in\{ \pm 1\},
$$

so $\gamma_{c}^{\prime}(x)$ is an integer and $A_{i_{1}, \ldots, i_{\mathrm{m}}}$ stays real. Thus $\gamma_{c}^{\prime}(x)$ jumps between even or odd integers in $\mathbb{Z}$, while the $\mathbb{Z}_{2}^{C}$-gauge transformation can be suitably formulated on a lattice. We can directly rewrite Eq. (2.23) on a simplicial complex or a triangulable spacetime manifold. Follow Ref. [4], we also define a covariant derivative with respect to $\mathbb{Z}_{2}^{C}$ :

$$
D_{\mu}^{c}:=\left(\partial_{\mu}-i g_{c} C_{\mu}\right) \text {. }
$$

We need to combine $\mathrm{U}(1)_{x_{(n)}}$-gauge transformation Eq. (2.16) and $\mathbb{Z}_{2}^{C}$-gauge transformation Eq. (2.23) to

$$
\begin{aligned}
A_{i_{1}, \ldots, i_{\mathrm{m}}} & \rightarrow e^{i \gamma_{c}(x)} A_{i_{1}, \ldots, i_{\mathrm{m}}}+\frac{1}{(\mathrm{~m} !) g}\left(D_{\left(i_{1}\right.}^{c} D_{i_{2}}^{c} \cdots D_{\left.i_{\mathrm{m}}\right)}^{c}\right)\left[\eta_{v}(x)\right], \\
C_{\nu} & \rightarrow C_{\nu}+\frac{1}{g_{c}} \partial_{\nu} \gamma_{c}(x) .
\end{aligned}
$$

Here $\quad\left(D_{\left(i_{1}\right.}^{c} D_{i_{2}}^{c} \cdots D_{\left.i_{\mathrm{m}}\right)}^{c}\right):=\left(D_{i_{1}}^{c} D_{i_{2}}^{c} \cdots D_{i_{\mathrm{m}}}^{c}+\right.$ $\left.D_{i_{2}}^{c} D_{i_{1}}^{c} \cdots D_{i_{\mathrm{m}}}^{c}+\cdots\right)$ contains the permutation ( $\mathrm{m}$ !)-terms, which means to be a symmetrization over the subindices under the lower bracket $\left(i_{1}, \ldots, i_{\mathrm{m}}\right)$.

We thus can promote the Abelian gauge field strength Eq. (2.20)'s $F_{\mu, v, i_{2}, \ldots, i_{\mathrm{m}}}$ into a non-Abelian gauge field strength $\hat{F}_{\mu, v, i_{2}, \ldots, i_{\mathrm{m}}}^{c}$ after gauging $\mathbb{Z}_{2}^{C}$ :

$$
\begin{aligned}
\hat{F}_{\mu, \nu, i_{2}, \ldots, i_{\mathrm{m}}}^{c} & :=D_{\mu}^{c} A_{v, i_{2}, \ldots, i_{\mathrm{m}}}-D_{\nu}^{c} A_{\mu, i_{2}, \ldots, i_{\mathrm{m}}} \\
& :=\left(\partial_{\mu}-i g_{c} C_{\mu}\right) A_{v, i_{2}, \ldots, i_{\mathrm{m}}}-\left(\partial_{\nu}-i g_{c} C_{\nu}\right) A_{\mu, i_{2}, \ldots, i_{\mathrm{m}}} .
\end{aligned}
$$

This $\hat{F}_{\mu, v, i_{2}, \ldots, i_{\mathrm{m}}}$ is covariant under the gauge transformation Eq. (2.26): $\hat{F}_{\mu, \nu, i_{2}, \ldots, i_{\mathrm{m}}}^{c} \rightarrow e^{i \gamma_{c}(x)} \hat{F}_{\mu, \nu, i_{2}, \ldots, i_{\mathrm{m}}}^{c}$. It is obvious that we can construct the gauge-invariant kinetic Lagrangian term

$$
\left|\hat{F}_{\mu, \nu, i_{2}, \ldots, i_{\mathrm{m}}}^{c}\right|^{2}:=\hat{F}_{\mu, \nu, i_{2}, \ldots, i_{\mathrm{m}}}^{c} \hat{F}^{\dagger c \mu, \nu, i_{2}, \ldots, i_{\mathrm{m}}},
$$

pairing $\hat{F}^{c}$ with its complex conjugation $\hat{F}^{\dagger c}$. We can propose a schematic path integral form:

$$
\begin{aligned}
\underset{\substack{\text { asym-BF } \\
\text { ak-sym-A }}}{\mathbf{x}}= & \int\left[\mathcal{D} A_{i_{1}, \ldots, i_{\mathrm{m}}}\right][\mathcal{D} B][\mathcal{D} C] \\
& \times \exp \left[i \int _ { M ^ { d + 1 } } \left(\left|\hat{F}_{\mu, v, i_{2}, \ldots, i_{\mathrm{m}}}^{c}\right|^{2} d^{d+1} x\right.\right. \\
& \left.\left.+\frac{2}{2 \pi} B d C\right)\right] \cdot \omega_{d+1}\left(\left\{C_{I}\right\}\right) .
\end{aligned}
$$

Based on the knowledge of the lower degree-1 polynomial gauge structure Eq. (1.1) and the higher-moment degree-(m 1) polynomial symmetry Eq. (1.7), we can denote the gauge structure for Eq. (2.28) as

$$
\left[\mathbb{Z}_{2}^{C} \ltimes\left(\mathrm{U}(1)_{\substack{\left(\begin{array}{c}
\mathrm{m}-1 \\
\mathrm{~m}-1
\end{array}\right) \\
\text { na }}}\right)\right] .
$$

If only the rank-m symmetry tensor gauge field $A_{i_{1}, \ldots, i_{\mathrm{m}}}$ is kept, then the lower degree polynomial symmetry in Eq. (1.7), say, $\mathrm{U}(1)_{x_{\left(\begin{array}{c}n \\ M\end{array}\right)}^{M}}$ for $0 \leqslant M \leqslant \mathrm{~m}-2$ would be neither a survived global symmetry nor a gauged symmetry.

More generally, we introduce the index $I$ for specifying the different copies/layers of tensor gauge theories,

$$
\begin{aligned}
\underset{\substack{\text { resym-BF } \\
\text { rk-sym- } A}}{\mathbf{a}_{I=1}} & \int\left(\prod_{I=1}^{N}\left[\mathcal{D} A_{I, i_{1}, \ldots, i_{\mathrm{m}}}\right]\left[\mathcal{D} B_{I}\right]\left[\mathcal{D} C_{I}\right]\right) \\
& \times \exp \left[i \int _ { M ^ { d + 1 } } d ^ { d + 1 } x \left(\sum_{I=1}^{N}\left|\hat{F}_{I, \mu, \nu, i_{2}, \ldots, i_{\mathrm{m}}}^{c}\right|^{2}\right.\right. \\
& \left.\left.+\frac{2}{2 \pi} \sum_{I=1}^{N} B_{I} d C_{I}\right)\right] \cdot \omega_{d+1}\left(\left\{C_{I}\right\}\right)
\end{aligned}
$$

where the level-2 BF theory is used to constrain the flat $C$ gauge field to be a $\mathbb{Z}_{2}$-valued 1 -form gauge field via a $\mathbb{Z}_{2}$-valued $(d-1)$-form gauge field $B$, based on the trick of Ref. [4]. The cocycle $\omega_{d+1} \in \mathrm{H}^{d+1}\left(\left(\mathbb{Z}_{2}^{C}\right)^{N}, \mathbb{R} / \mathbb{Z}\right)$ is a group cohomology data [14] where we apply its continuum field theory formulation [16-19] (see the overview [4]). The cocycle $\omega_{d+1}$ couples different copies/layers of tensor gauge theories together, which can be viewed as interlayer interaction effects.

Above we formulate a general degree polynomial as a higher-moment global symmetry and construct the field strength $F$ for the gauge theory. Our theory presented in Sec. II A is general. Let us take two special examples in the next subsections, for a polynomial of degree 1 in Sec. II B and degree 2 in Sec. II C.

\section{B. Polynomial with degree 1: Vector symmetry}

For a vector global symmetry of a polynomial with degree 1 , the symmetry transformation on the scalar field and the invariant quantity under this transformation are as follows:

$$
\Phi \rightarrow e^{i Q(x)} \Phi=e^{i\left(\Lambda_{i} x_{i}+\Lambda_{0}\right)} \Phi,
$$

$\log \Phi \rightarrow \log \Phi+i Q(x)=\log \Phi+i\left(\Lambda_{i} x_{i}+\Lambda_{0}\right)$,

$$
\begin{aligned}
\partial_{x_{i}} \partial_{x_{j}} \log \Phi & =\frac{P_{x_{i}, x_{j}}\left(\Phi, \partial \Phi, \partial^{2} \Phi\right)}{\Phi^{2}} \\
& =\frac{\Phi \partial_{x_{i}} \partial_{x_{j}} \Phi-\left(\partial_{x_{i}} \Phi\right)\left(\partial_{x_{j}} \Phi\right)}{\Phi^{2}} \rightarrow \partial_{x_{i}} \partial_{x_{j}} \log \Phi
\end{aligned}
$$

$$
\partial_{i} \partial_{j} \log \Phi=\frac{\Phi \partial_{i} \partial_{j} \Phi-\left(\partial_{i} \Phi\right)\left(\partial_{j} \Phi\right)}{\Phi^{2}} .
$$

In the last line, we simply shorthand $x_{i}, x_{j}$ as $i, j$. To gauge,we rewrite $Q(x)$ as a local gauge parameter $\eta(x)$,

$$
\partial_{i} \partial_{j} \log \Phi \rightarrow \partial_{i} \partial_{j} \log \Phi+i \partial_{i} \partial_{j} \eta(x) .
$$


This implies that we can write the gauge covariant operator $D_{i, j}[\{\Phi\}]$ via

$$
\begin{aligned}
& P_{i, j}\left(\Phi, \partial \Phi, \partial^{2} \Phi\right) \\
&:=\left[\Phi \partial_{i} \partial_{j} \Phi-\left(\partial_{i} \Phi\right)\left(\partial_{j} \Phi\right)\right] \\
& \rightarrow e^{i 2 \eta(x)}\left[P_{i, j}\left(\Phi, \partial \Phi, \partial^{2} \Phi\right)+i \partial_{i} \partial_{j} \eta(x)\right] . \\
& A_{i, j} \rightarrow A_{i, j}+\frac{1}{g} \partial_{i} \partial_{j} \eta . \\
& D_{i, j}[\{\Phi\}]:=P_{i, j}\left(\Phi, \partial \Phi, \partial^{2} \Phi\right)-i g A_{i, j} \Phi^{2} \\
&=\left[\Phi \partial_{i} \partial_{j} \Phi-\left(\partial_{i} \Phi\right)\left(\partial_{j} \Phi\right)-i g A_{i, j} \Phi^{2}\right] .
\end{aligned}
$$

Physically we may define the symmetry transformation

$$
e^{i \Lambda \cdot x}=e^{i 2 \pi\left(\lambda^{-1}\right) \cdot x} .
$$

So the $\lambda$ is a vector of an effective wavelength while $\vec{\Lambda}=$ $|\Lambda| \hat{\Lambda}=\frac{2 \pi}{|\lambda|} \hat{\lambda}$, with the unit vector $\hat{\Lambda}=\hat{\lambda}$. So a gauge-invariant term can be $\left|D_{i, j}[\{\Phi\}]\right|^{2}$.

See another route of pursuit for the vector global symmetry recently proposed by Seiberg [38].

\section{Polynomial with degree 2: Higher-moment symmetry}

For a vector global symmetry of a polynomial with degree 2 , the symmetry transformation on the scalar field and the invariant quantity under this transformation are as follows:

$$
\Phi \rightarrow e^{i Q(x)} \Phi=e^{i\left(\Lambda_{i, j} x_{i} x_{j}+\Lambda_{i} x_{i}+\Lambda_{0}\right)} \Phi,
$$

$\log \Phi \rightarrow \log \Phi+i Q(x)=\log \Phi+i\left(\Lambda_{i, j} x_{i} x_{j}+\Lambda_{i} x_{i}+\Lambda_{0}\right)$,

$$
\begin{aligned}
\partial_{i} \partial_{j} \partial_{k} \log \Phi & =\frac{P_{i, j, k}\left(\Phi, \ldots, \partial^{3} \Phi\right)}{\Phi^{3}} \\
& :=\frac{\Phi^{2}\left(\partial_{i} \partial_{j} \partial_{k} \Phi\right)-\Phi\left(\left(\partial_{k} \Phi\right)\left(\partial_{i} \partial_{j} \Phi\right)+\left(\partial_{i} \Phi\right)\left(\partial_{j} \partial_{k} \Phi\right)+\left(\partial_{j} \Phi\right)\left(\partial_{i} \partial_{k} \Phi\right)\right)+2\left[\left(\partial_{i} \Phi\right)\left(\partial_{j} \Phi\right)\left(\partial_{k} \Phi\right)\right]}{\Phi^{3}} . \\
& :=\frac{\Phi^{2}\left(\partial_{i} \partial_{j} \partial_{k} \Phi\right)-3 \Phi\left(\partial_{(k} \Phi \partial_{i} \partial_{j)} \Phi\right)+2\left(\partial_{i} \Phi\right)\left(\partial_{j} \Phi\right)\left(\partial_{k} \Phi\right)}{\Phi^{3}} .
\end{aligned}
$$

This implies that we can write the gauge covariant operator $D_{i, j, k}[\{\Phi\}]$ via the following:

$$
\begin{aligned}
P_{i, j, k}\left(\Phi, \ldots, \partial^{3} \Phi\right) & :=\Phi^{2}\left(\partial_{i} \partial_{j} \partial_{k} \Phi\right)-\Phi\left(\left(\partial_{k} \Phi\right)\left(\partial_{i} \partial_{j} \Phi\right)+\left(\partial_{i} \Phi\right)\left(\partial_{j} \partial_{k} \Phi\right)+\left(\partial_{j} \Phi\right)\left(\partial_{i} \partial_{k} \Phi\right)\right)+2\left(\partial_{i} \Phi\right)\left(\partial_{j} \Phi\right)\left(\partial_{k} \Phi\right) \\
& :=\Phi^{2}\left(\partial_{i} \partial_{j} \partial_{k} \Phi\right)-3 \Phi\left(\partial_{(k} \Phi \partial_{i} \partial_{j} \Phi\right)+2\left(\partial_{i} \Phi\right)\left(\partial_{j} \Phi\right)\left(\partial_{k} \Phi\right) \rightarrow e^{i 3 \eta(x)}\left(P_{i, j, k}\left(\Phi, \ldots, \partial^{3} \Phi\right)+i \partial_{i} \partial_{j} \partial_{k} \eta(x)\right) .
\end{aligned}
$$

$$
\begin{gathered}
A_{i, j, k} \rightarrow A_{i, j, k}+\frac{1}{g} \partial_{i} \partial_{j} \partial_{k} \eta . \\
D_{i, j, k}[\{\Phi\}]:=P_{i, j, k}\left(\Phi, \ldots, \partial^{3} \Phi\right)-i g A_{i, j, k} \Phi^{3} .
\end{gathered}
$$

In the above we use the symmetrized tensor notation: $T_{\left(i_{1} i_{2} \cdots i_{k}\right)}=\frac{1}{k !} \sum_{\sigma \in \mathfrak{S}_{k}} T_{i_{\sigma} i_{\sigma 2} \cdots i_{\sigma k}}$, with parentheses $(i j k)$ around the indices being symmetrized. The $\mathfrak{S}_{k}$ is the symmetric group of $k$ symbols. So a gauge-invariant term can be $\left|D_{i, j, k}[\{\Phi\}]\right|^{2}$.

\section{VECTOR CHARGE, TENSOR CHARGE, AND GENERAL HIGHER-MOMENT SYMMETRY}

\section{A. Vector charge}

We may also consider more general higher-moment conservation laws with a set of number $r$ fields $\Phi_{1}, \ldots, \Phi_{r}$ and gauge transformations

$$
\Phi_{I} \rightarrow e^{i Q_{I}(x)} \Phi_{I}
$$

where

$$
\begin{aligned}
Q_{I} \in V_{I} \subset \oplus_{I=1}^{r} V_{I} & =V \subset \mathbb{R}^{r} \otimes \mathbb{R}\left[x_{1}, \ldots, x_{n}\right] \\
& =\oplus_{I=1}^{r} \mathbb{R}\left[x_{1}, \ldots, x_{n}\right] .
\end{aligned}
$$

The $V_{I}$ denotes the vector space where the polynomial $Q_{I}=$ $Q_{I}(x)$ lives. A special case is $V_{I}=\mathbb{R}\left[x_{1}, \ldots, x_{n}\right]$. For this vector space $\mathbb{R}\left[x_{1}, \ldots, x_{n}\right]$, we have the vector addition in terms of the polynomial addition, while we have the scalar multiplication in terms of the scalar in the real number $\mathbb{R}$ multiplying by the polynomial.

Note that the full vector space $V$ is fully characterized by another vector space $D$ of differential operators, which annihilate the space $V$. This space $D_{I}$ is not finite dimensional, but we may take a finite dimensional subspace $\tilde{D}$ generating the vector space $D$. Namely, we can take differential operators $\mathfrak{D}_{J}^{I}$ such that

$$
\sum_{I} \mathfrak{D}_{J}^{I} Q_{I}=0
$$

for any $Q_{I} \in V$. If

$$
\sum_{I} \mathfrak{D}^{I} Q_{I}=0
$$

for any $Q$ we have

$$
\mathfrak{D}^{I}=\sum a^{J} \mathfrak{D}_{J}^{I}
$$

for some $a^{J}$. In the previous example, we took homogeneous polynomials of degree $m$.

Following the same logic, we may show that each elements in $\tilde{D}$ gives an invariant field strength. To gauge this symmetry, we need to introduce gauge fields which one to one correspond to elements in $\tilde{D}$. The gauge transformation law is 
transparent: they are just differential operators $\mathfrak{D}_{J}^{I}$ in $\tilde{D}$ acting on the gauge variational parameter, say, $\eta_{I}$.

Let us be more concrete: under the gauge transformation $\Phi_{I} \rightarrow e^{i Q_{I}(x)} \Phi_{I}$, we have $\log \Phi_{I} \rightarrow \log \Phi_{I}+i Q_{I}(x)$ and

$$
\sum_{I} \mathfrak{D}_{J}^{I} \log \Phi_{I} \rightarrow \mathfrak{D}_{J} \log \Phi_{I}
$$

as $\sum_{I} \mathfrak{D}_{J}^{I} Q_{I}=0$.

We can compute

$$
\sum_{I} \mathfrak{D}_{J}^{I} \log \Phi_{I}=\frac{P_{J}}{\mathcal{Q}_{J}}
$$

where $P_{J}$ is a polynomial in fields $\Phi_{I}$ and their derivatives, and $\mathcal{Q}_{J}$ is a monomial in $\Phi_{I}$. Namely, $\mathcal{Q}_{J}=\prod_{I}\left(\Phi_{I}\right)^{\left(n_{J}^{I}\right)}$ is a product of $\Phi_{I}$, where $n_{J}^{I}$ is an integer power of some $\Phi_{I}$.

Therefore we can see that the denominator $\mathcal{Q}_{J}$ as a monomial transforms in the following way and the same for the numerator $P_{J}$ :

$$
\begin{gathered}
\mathcal{Q}_{J} \rightarrow e^{i \zeta_{J}(x)} \mathcal{Q}_{J}, \\
P_{J} \rightarrow e^{i \zeta_{J}(x)} P_{J},
\end{gathered}
$$

for some polynomial $\zeta_{J}=\zeta_{J}(x)$ depending on our data. Each of these $P_{J}$ corresponds to a covariant derivative under a general gauge transformation

$$
\Phi_{I} \rightarrow e^{i \eta_{I}(x)} \Phi_{I}
$$

We can compute

$$
\sum_{I} \mathfrak{D}_{J}^{I} \log \Phi_{I}=\frac{P_{J}}{\mathcal{Q}_{J}}+i \sum_{I} \mathfrak{D}_{J}^{I} \eta_{I},
$$

hence $P_{J}$ transform as

$$
P_{J} \rightarrow e^{i \zeta_{J}(x)} P_{J}+i \sum_{I} \mathfrak{D}_{J}^{I} \eta_{I} \mathcal{Q}_{J}
$$

Therefore we introduce a covariant derivative

$$
D_{J}\left[\left\{\Phi_{I}\right\}\right]:=R_{J} \equiv P_{J}-i g A_{J} \mathcal{Q}_{J},
$$

where under the gauge transformation of $\Phi_{I} \rightarrow e^{i \eta_{I}(x)} \Phi_{I}$, we have the gauge transformation of

$$
A_{J} \rightarrow A_{J}+\frac{1}{g} \sum_{I} \mathfrak{D}_{J}^{I} \eta_{I} .
$$

Then $R_{J}$ transforms as $R_{J} \rightarrow e^{i \zeta_{J}(x)} R_{J}$. We construct the gauge-invariant matter-gauge field interaction term in the Lagrangian

$$
\begin{aligned}
\left|D_{J}\left[\left\{\Phi_{I}\right\}\right]\right|^{2} & :=\left|R_{J}\right|^{2}:=\left(R_{J}\right)\left(R_{J}^{\dagger}\right) \\
& =\left(P_{J}-i g A_{J} \mathcal{Q}_{J}\right)\left(P_{J}^{\dagger}+i g A_{J} \mathcal{Q}_{J}^{\dagger}\right)
\end{aligned}
$$

\section{B. Tensor charge}

It is embarrassingly easy to generalize to this tensor-index complex scalar field for the global symmetry transformation:

$$
\Phi_{I_{1}, \ldots, I_{M}} \rightarrow e^{i Q_{I_{1}, \ldots, I_{M}}(x)} \Phi_{I_{1}, \ldots, I_{M}},
$$

and the gauge symmetry transformation with a local dependent gauge parameter $\eta_{I_{1}, \ldots, I_{M}}(x)$ :

$$
\Phi_{I_{1}, \ldots, I_{M}} \rightarrow e^{i \eta_{I_{1}, \ldots, I_{M}}(x)} \Phi_{I_{1}, \ldots, I_{M}} .
$$

Nonetheless, we just need to give a one-to-one map $\left(I_{1}, \ldots, I_{M}\right) \rightarrow I$, so the tensor indices can be mapped to a vector index, which transforms the above two equations into

$$
\Phi_{I} \rightarrow e^{i \alpha_{I}(x)} \Phi_{I}
$$

and

$$
\Phi_{I} \rightarrow e^{i \eta_{I}(x)} \Phi_{I},
$$

respectively. Thus the tensor charge higher-moment global symmetry can be treated as the same way as the vector charge higher-moment global symmetry in Sec. III B under the oneto-one map $\left(I_{1}, \ldots, I_{M}\right) \rightarrow I$.

\section{Example 1: Vector charge with an exclusive degree-1 polynomial}

As a special case, we can recover the vector charged tensor gauge theory by taking the vector space of degree-1 polynomial

$$
V_{I}=\left\{1, x_{I-1}, x_{I+1}\right\},
$$

which means that it is spanned by the vectors of $1, x_{I-1}$, and $x_{I+1}$. The global symmetry acts as

$$
\begin{aligned}
\Phi_{I}(x) \rightarrow e^{i Q_{I}(x)} \Phi_{I}(x) & =e^{i Q_{I}\left(x_{I-1}, x_{I+1}\right)} \Phi_{I}(x) \\
& =e^{i\left(\Lambda_{I+1} x_{I-1}-\Lambda_{I-1} x_{I+1}+\Lambda_{0}\right)} \Phi_{I}(x) .
\end{aligned}
$$

Here we may define

$$
\begin{aligned}
x_{I+l^{\prime}} & :=x_{I+l^{\prime}} \bmod n, \text { with the subindex where } I+l^{\prime} \\
& :=I+l^{\prime} \bmod n .
\end{aligned}
$$

In fact, our specific example here is a generalization of one example in Pretko [27]. ${ }^{7}$ We may call this type of $Q_{I}(x)=$ $Q_{I}\left(x_{I-1}, x_{I+1}\right)$ an exclusive polynomial which the $Q_{I}$ excludes the $x_{I}$ dependency, thus it is $x_{I}$ independent.

The vector space $V$ is fully characterized by another vector space $D$ of differential operators, which annihilate $V$ by differential. This space $D_{I}$ is not finite dimensional, but we may take a finite dimensional subspace $\tilde{D}$ generating the vector space $D$, here $^{8}$

$$
\tilde{D}=\left\{\partial_{(i=I)} \Phi_{I}, \Phi_{J} \partial_{(j=J)} \Phi_{K}+\Phi_{K} \partial_{(k=K)} \Phi_{J}\right\},
$$

where $j=J$ and $k=K$ are related by

$$
j=k \pm 1 \bmod n \text {. }
$$

Here the spacetime index $(i, j, k, \ldots)$ and the internal vector index $(I, J, K, \ldots)$ of $\Phi$ fields are locked.

Furthermore, we can effectively construct the gauge theory explicitly, given by the rule of a gauge principle. For this

\footnotetext{
${ }^{7}$ Our result in Eq. (3.19) generalizes Pretko's

$$
\Phi_{I}(x) \rightarrow e^{i \sum_{J, K} \varepsilon_{I J K} \Lambda_{J} x_{K}} \Phi_{I}(x),
$$
}

where $\varepsilon_{I J K}=\varepsilon^{I J K}$ is just a Levi-Civita symbol, or a so-called alternating tensor.

${ }^{8}$ In this notation below $\Phi_{I} \rightarrow e^{i Q_{I}(x)} \Phi_{I}=e^{i Q_{I}\left(x_{I-1}, x_{I+1}\right)} \Phi_{I}$, we focus just on the dependence of $x$ only on $Q_{I}(x)$, not $\Phi_{I}$. 
special case, we can recover the vector charged tensor gauge theory and covariant derivatives:

$$
\begin{aligned}
& \left\{\partial_{(i=I)} \Phi_{I}-i g A_{I} \Phi_{I}, \quad \Phi_{J} \partial_{(j=J)} \Phi_{K}+\Phi_{K} \partial_{(k=K)} \Phi_{J}\right. \\
& \left.\quad-i g A_{(j=J)(k=K)} \Phi_{J} \Phi_{K}\right\} .
\end{aligned}
$$

In short, by locking $i=I, j=J$ and $k=K$, we simply write

$$
\left\{\partial_{i} \Phi_{i}-i g A_{i} \Phi_{i}, \quad \Phi_{j} \partial_{j} \Phi_{k}+\Phi_{k} \partial_{k} \Phi_{j}-i g A_{j k} \Phi_{J} \Phi_{K}\right\} .
$$

We can effectively construct everything explicitly, given the rule of gauge transformations:

$$
\begin{gathered}
\Phi_{I} \rightarrow e^{i \eta_{I}(x)} \Phi_{I}, \\
A_{j k} \rightarrow A_{j k}+\frac{1}{g}\left(\partial_{j} \eta_{k}+\partial_{k} \eta_{j}\right) .
\end{gathered}
$$

\section{Example 2: Vector charge with an inclusive degree-1 polynomial}

Let us consider another simple example: Given fields $\Phi_{1}$, $\Phi_{2}, \ldots$, and the higher-moment global symmetry:

$$
\Phi_{i} \rightarrow e^{i \Lambda x_{i}} \Phi_{i}
$$

We may call this type of $Q_{I}(x)=Q_{I}\left(x_{I}\right)$ an inclusive polynomial, in which the $Q_{I}$ include only the $x_{I}$ dependency. We have an invariant Lagrangian term $\left|\Phi_{2} \partial_{1} \Phi_{1}-\Phi_{1} \partial_{2} \Phi_{2}\right|^{2}$. We can introduce a tensor connection field $A_{12}$, and then the covariant derivative type of Lagrangian term $\mid \Phi_{2} \partial_{1} \Phi_{1}-\Phi_{1} \partial_{2} \Phi_{2}-$ $\left.i_{g} A_{12} \Phi_{1} \Phi_{2}\right|^{2}$. More generally, we have

$$
\left|\Phi_{i} \partial_{j} \Phi_{j}-\Phi_{j} \partial_{i} \Phi_{i}-i g A_{i j} \Phi_{i} \Phi_{j}\right|^{2}
$$

invariant under a general gauge transformation

$$
\begin{gathered}
\Phi_{j} \rightarrow e^{i \eta_{j}(x)} \Phi_{j}, \\
A_{i j} \rightarrow A_{i j}+\frac{1}{g}\left(\partial_{i} \eta_{i}-\partial_{j} \eta_{j}\right),
\end{gathered}
$$

where $i, j$ can be any coordinate since we have this specific global symmetry: $\Phi_{i} \rightarrow e^{i \Lambda x_{i}} \Phi_{i}$ for any $x_{i}$. Importantly, the generic gauge field $A_{i j}$ is not symmetric under $i \leftrightarrow j .{ }^{9}$ This example reveals that the generic higher-moment global symmetry for a vector-index charge field, after gauging, does not yield a symmetric tensor gauge field.

\footnotetext{
${ }^{9} A_{i j}$ can be made symmetric if we revise the transformation law, for a specific pair of $(i, j)$, such that $\Phi_{i} \rightarrow e^{i \Lambda x_{i}} \Phi_{i}$ and $\Phi_{j} \rightarrow e^{-i \Lambda x_{j}} \Phi_{j}$, so that the Lagrangian term

$$
\left|\Phi_{i} \partial_{j} \Phi_{j}+\Phi_{j} \partial_{i} \Phi_{i}-i g A_{i j} \Phi_{i} \Phi_{j}\right|^{2}
$$
}

is invariant under a general gauge transformation

$$
\Phi_{j} \rightarrow e^{i \eta_{j}(x)} \Phi_{j}, \quad A_{i j} \rightarrow A_{i j}+\frac{1}{g}\left(\partial_{i} \eta_{i}+\partial_{j} \eta_{j}\right) .
$$

\section{E. Example 3: Vector charge with a mixed degree-1 polynomial}

Consider the vector-index charge fields: $\Phi_{j}$. Consider the higher-moment global symmetry:

$$
\Phi_{j} \rightarrow e^{i \Lambda x_{1}} \Phi_{j}
$$

where $j=1,2, \ldots$ We have an invariant Lagrangian term $\left|\Phi_{2} \partial_{1} \Phi_{1}-\Phi_{1} \partial_{1} \Phi_{2}\right|^{2}$ and other invariant Lagrangian terms $\left|\Phi_{j} \partial_{1} \Phi_{i}-\Phi_{i} \partial_{1} \Phi_{j}\right|^{2}$. We can introduce a tensor gauge connection field $A_{i j}$, and then the covariant derivative

$$
\Phi_{j} \partial_{1} \Phi_{i}-\Phi_{i} \partial_{1} \Phi_{j}-i A_{i j} \Phi_{i} \Phi_{j}
$$

is invariant under a general gauge transformation

$$
\begin{gathered}
\Phi_{j} \rightarrow e^{i \eta_{j}(x)} \Phi_{j}, \\
A_{i j} \rightarrow A_{i j}+\left(\partial_{1} \eta_{i}-\partial_{1} \eta_{j}\right) .
\end{gathered}
$$

Again $i, j$ can be any coordinate since we have this specific global symmetry: $\Phi_{i} \rightarrow e^{i \Lambda x_{1}} \Phi_{i}$ for any $x_{i}$. Importantly, similarly to our results in Sec. III E, the generic gauge field $A_{i j}$ is not symmetric under $i \leftrightarrow j$

\section{GENERALIZING NOETHER'S THEOREM FOR HIGHER-MOMENT GLOBAL SYMMETRY}

Suppose we have a set of $r$ fields $\Phi_{I}(1 \leqslant I \leqslant r)$, a Lagrangian term $L$ which is invariant under a global transformations $\Phi_{I} \rightarrow e^{i Q_{I}(x)} \Phi_{I}$ where $\left(Q_{1}, \ldots, Q_{r}\right) \in V \subset$ $\bigoplus_{I=1}^{r} \mathbb{R}\left[x_{1}, \ldots, x_{d}\right]$ is a specified vector space of allowed polynomials.

Noether's theorem guarantees that we have a conserved current corresponding to each global symmetry. Suppose the constant $\mathrm{U}(1)$ transformation for each field is a global symmetry, Noether's theorem says that we have a one form current

$$
j_{I}=j_{I \mu} d x^{\mu},
$$

such that under the general infinitesimal variation

$$
\Phi_{I} \rightarrow e^{i \epsilon \alpha_{I}(x)} \Phi_{I},
$$

the Lagrangian density transforms as

$$
\delta \mathcal{L}=\epsilon \alpha_{I} \wedge \star j_{I} .
$$

The $\epsilon$ is an infinitesimal variational parameter. Here $\rho_{I}=j_{I 0}$ is the spatial density of the conserved charge.

Now let us take $\alpha_{I}=Q_{I}(x)$ as the higher-moment global symmetry polynomial for

$$
\Phi_{I} \rightarrow e^{i Q_{I}(x)} \Phi_{I} .
$$

As we said earlier, we see that $\int \delta \mathcal{L}=0$, and therefore

$$
\int_{\text {space }} \sum_{I} \rho_{I} Q_{I}=\int_{\text {space }} \sum_{I} j_{I 0} Q_{I}
$$

is a conserved charge. That is, we have a conserved charge for each of the global symmetry we have, and their number is precisely the dimension of the vector space $V$ we started with.

By doing the above calculation, we need to be careful about the boundary conditions of the space manifold or the infinite faraway field configurations of the space manifold. In most cases, we can assume that the density of field configurations decays sharply at the infinite faraway. 
Let us take $Q_{I}(x)$ is a polynomial over the spatial coordinates. Here are some examples:

(1) For a single field $\Phi$, when $Q(x)=\Lambda$ is a constant, we have the usual Noether's theorem for the ordinary U(1) global symmetry, with a conserved charge:

$$
\int_{\text {space }} \rho=\int_{\text {space }} j_{0} .
$$

(2) For a single field $\Phi$, when $Q(x)=\Lambda_{i} x_{i}+\Lambda_{0}$ is a linear degree-1 polynomial, we have a conservation theorem for the vector $U(1)$ global symmetry. This coincides with an example of Pretko [24].

$$
\begin{aligned}
\Lambda_{0}, \mathrm{~s}: \int_{\text {space }} \rho & =\int_{\text {space }} j_{0} . \\
\Lambda_{i} \text { 's : } \int_{\text {space }} \rho x_{i} & =\int_{\text {space }} j_{0} x_{i} .
\end{aligned}
$$

There are the same number of conserved charges as the dimensions of the vector space (the independent parameters $\Lambda_{0}$ and $\Lambda_{i}$ of the degree-1 polynomials).

(3) For a single field $\Phi$, when we follow Eq. (2.2) with

$Q(x):=\left(\Lambda_{i_{1}, \ldots, i_{\mathrm{m}-1}} x_{i_{1}} \ldots x_{i_{\mathrm{m}-1}}+\cdots+\Lambda_{i, j} x_{i} x_{j}+\Lambda_{i} x_{i}+\Lambda_{0}\right)$

of a degree- $(\mathrm{m}-1)$ polynomial, we have a conservation theorem for all independent $\Lambda_{i_{1}, \ldots, i_{k}}$

$$
\Lambda_{i_{1}, \ldots, i_{k}} \text { s } \mathrm{s}: \int_{\text {space }}(\rho) \cdot\left(x_{1} \ldots x_{k}\right)=\int_{\text {space }}\left(j_{0}\right) \cdot\left(x_{1} \ldots x_{k}\right)
$$

for the higher-moment $\mathrm{U}(1)$ global symmetry. There are the same number of conserved charges as the dimensions of the vector space (the independent parameters $\Lambda_{i_{1}, \ldots, i_{k}}$ ).

(4) For a vector-index field $\Phi_{I}$, with

$$
\begin{aligned}
Q_{I}(x):= & \left(\Lambda_{I ; i_{1}, \ldots, i_{\mathrm{m}-1}} x_{i_{1}} \ldots x_{i_{\mathrm{m}-1}}\right. \\
& \left.+\cdots+\Lambda_{I ; i, j} x_{i} x_{j}+\Lambda_{I ; i} x_{i}+\Lambda_{I ; 0}\right) .
\end{aligned}
$$

There are the same number of conserved charges as the dimensions of the vector space (the independent parameters $\Lambda_{I ; i_{1}, \ldots, i_{k}}$.

(5) For a tensor-index field $\Phi_{I_{1}, \ldots, I_{M}}$, we can map to a vector-index field $\Phi_{I}$ by a one-to-one map $\left(I_{1}, \ldots, I_{M}\right) \rightarrow I$, thus the result follows from the previous remark.

In all cases, if we have additional constraints (such as from the constraint of field strength, say, the electric tensor in Ref. [24] to be traceless, say, $\tilde{\mathrm{E}}_{j}^{j}=0$ for $\tilde{\mathrm{E}}_{i j}=-\partial_{0} A_{i j}+$ $\partial_{i} \partial_{j} A_{0}=-\partial_{t} A_{i j}+\partial_{i} \partial_{j} A_{0}$ for the notation in Ref. [4]), then we have additional conservation laws, not accounted for by the previously counted number of conserved laws as the dimensions of the vector space of $Q_{I}(x)$.

\section{CONCLUSION AND RELATIONS TO ALGEBRAIC GEOMETRY}

In this section, we bridge the relations between our theories (both the matter or the gauge theories) by physics construction and the algebraic geometry in mathematics. We conclude with some final comments.

\section{A. Algebraic (affine) variety and subvariety}

In mathematics, the polynomials are related to geometric objects called the algebraic variety. More precisely, (affine) varieties are defined as the solutions of polynomial equations. The morphisms between them are maps defined by polynomials. Here we review their basic definitions for both physicists and mathematicians:

Definition 1. An affine algebraic variety over real numbers $\mathbb{R}$ is the zero-locus in the affine space $\mathbb{R}^{n}$ of some finite family of polynomials of $n$ variables with coefficients in $\mathbb{R}$.

Definition 2. A morphism, or a regular map, of affine varieties is a function between affine varieties which is polynomial in each coordinate: more precisely, for affine varieties $V \subseteq \mathbb{R}^{n}$ and $W \subseteq \mathbb{R}^{m}$, a morphism from $\mathrm{V}$ to $\mathrm{W}$ is a map $\phi: V \longrightarrow W$ of the form $\phi\left(a_{1}, \ldots, a_{n}\right)=\left(f_{1}\left(a_{1}, \ldots, a_{n}\right), \ldots, f_{m}\left(a_{1}, \ldots, a_{n}\right)\right)$, where $f_{i} \in \mathbb{R}\left[X_{1}, \ldots, X_{n}\right]$ for each $i=1, \ldots, m$. Here $\left(a_{1}, \ldots, a_{n}\right) \in V \subseteq \mathbb{R}^{n}$ and $\phi\left(a_{1}, \ldots, a_{n}\right) \in W \subseteq \mathbb{R}^{m}$.

Definition 3. Given two affine varieties $V, W \subseteq \mathbb{R}^{n}, \mathrm{~V}$ is called a subvariety of $\mathrm{W}$, if $V \subseteq W$ as subsets of $\mathbb{R}^{n}$.

Definition 4. Two affine varieties $\mathrm{V}$ and $\mathrm{W}$ are isomorphic if there exist morphisms $\phi: V \longrightarrow W$ and $\psi: W \longrightarrow V$ such that $\psi \circ \phi=i d_{V}$ and $\phi \circ \psi=i d_{W}$, where $i d$ is the identity map.

For example, $x^{2}+y^{2}=1$ defines the unit circle and $\frac{t^{2}}{4}+$ $w^{2}=1$ defines an ellipse, both on the plane $\mathbb{R}^{2}$. There is a polynomial map $t \rightarrow 2 x, w \rightarrow y$ which identifies circle and ellipse, with the inverse given by a rescaling $x \rightarrow t / 2, y \rightarrow$ $w$, and therefore in algebraic geometry they are isomorphic.

In our setting, the higher-moment global symmetry transformations are given by polynomials on the space (here we focus on the Cartesian $\mathbb{R}^{n}$ or $\mathbb{R}^{d}$ defined earlier), and the contours (or constant hypersurfaces) are given by solutions of polynomials: they are subvarieties of our space (here on the Cartesian $\mathbb{R}^{n}$ or $\mathbb{R}^{d}$ ).

We should mention that Refs. [8,9] has a different look on the algebraic variety: the topological degeneracy of the gapped fractonic topologically ordered state in Ref. [9] are encoded also in an algebraic variety, which is defined by the common zeros of a set of polynomials over a finite field.

In contrast, the algebraic variety in our case is a way to organize the data of generalized higher-moment or subdimensional polynomial global symmetry or its gauge theory. The use of algebraic variety for our wide classes of theories does not require being a gapped (fractonic) topological order. Our theories include gapless or gapped theories.

\section{B. From higher-moment to subdimensional or subsystem polynomial global symmetry}

Let us relate the algebraic (affine) variety and subvariety in Sec. V A to the patterns of polynomials in the higher-moment or subdimensional or subsystem polynomial global symmetry or their gauge theories. The studies of subdimensional or subsystem global symmetries can be traced back to as early as Refs. [40,41] in the condensed matter literature. Here we generalize the concept to study the subdimensional or subsystem polynomial global symmetry For instance, subsystem global symmetry can act on lines $[42,43]$ or planes $[9,44,45]$, for the bulk of $2+1 \mathrm{D}$ systems $[42,43]$ or $3+1 \mathrm{D}$ systems $[9,42,45]$. 
(1) From a degree-1 higher-moment symmetry to a degree0 ordinary global symmetry in subdimensions: Recall the degree-1 polynomial global symmetry of Eq. (2.31), $\Phi \rightarrow$ $e^{i Q(x)} \Phi=e^{i\left(\Lambda_{i} x_{i}+\Lambda_{0}\right)} \Phi$ acting on the matter field $\Phi$ on $\mathbb{R}^{n}$. We can relate this degree-1 polynomial global symmetry to a degree- 0 ordinary global symmetry by taking the constant surface solution of

$$
\left(\Lambda_{i} x_{i}+\Lambda_{0}\right)=\Lambda_{\text {constant }}
$$

for a certain $(n-1) \mathrm{D}$ subdimensional space (e.g., plane) of $x_{i} \in \mathbb{R}^{n}$.

(2) From a degree-2 higher-moment symmetry to a degree-1 higher-moment or degree-0 ordinary global symmetry: Recall the degree-2 polynomial global symmetry of Eq. (2.40), $\Phi \rightarrow e^{i Q(x)} \Phi=e^{i\left(\Lambda_{i, j} x_{i} x_{j}+\Lambda_{i} x_{i}+\Lambda_{0}\right)} \Phi$ acting on the matter field $\Phi$ on $\mathbb{R}^{n}$. We can relate this degree- 2 polynomial global symmetry to a degree-1 symmetry by restricting to an appropriate constant $x_{i}=c_{i}$ space for some specific $x_{i}$. For example, we have

$$
\left.\left(\Lambda_{i, j} x_{i} x_{j}+\Lambda_{i} x_{i}+\Lambda_{0}\right)\right|_{\left(x_{i}=c_{i}\right)}=\left(\Lambda_{i, j} c_{i} x_{j}+\Lambda_{i} c_{i}+\Lambda_{0}\right)
$$

for a certain $(n-1) \mathrm{D}$ subdimensional space of $x_{i} \in \mathbb{R}^{n}$. Moreover, we can reduce to a degree- 0 ordinary global symmetry, if there is an intersecting subspace between the constant spaces of $x_{i}=c_{i}$ and $x_{j}=c_{j}$, e.g.,

$\left.\left(\Lambda_{i, j} x_{i} x_{j}+\Lambda_{i} x_{i}+\Lambda_{0}\right)\right|_{\left(x_{i}=c_{i}, x_{j}=c_{j}\right)}=\left(\Lambda_{i, j} c_{i} c_{j}+\Lambda_{i} c_{i}+\Lambda_{0}\right)$.

Depending on the $\Lambda_{i, j}$ and $\Lambda_{i}$, there could be a different constant surface by solving the polynomial with a different set of constraints.

For example, given a two-variable quadratic equation

$$
\begin{aligned}
Q(x):= & Q\left(x_{1}, x_{2}\right)=\Lambda_{1,1}\left(x_{1}\right)^{2}+\Lambda_{1,2} x_{1} x_{2}+\Lambda_{2,2}\left(x_{2}\right)^{2} \\
& +\Lambda_{1} x_{1}+\Lambda_{2} x_{2}+\Lambda_{0},
\end{aligned}
$$

we can solve the constant space to be an ellipse, a parabola, or a hyperbola, and also possibly a circle, a line, or two crossing lines, etc. The solution is a quadratic algebraic curves through the well-known conic section. In other words, if we apply the degree-2 polynomial global symmetry of $e^{i Q(x)}$ under Eq. (5.1), we can find the degree-2 polynomial global symmetry on the $\mathbb{R}^{n}$ reduced to the ordinary degree- 0 global symmetry on the algebraic curves (an ellipse, a parabola or a hyperbola, etc.) through the well-known conic section.

(3) From a degree-( $(\mathrm{m}-1)$ higher-moment symmetry to a subdimensional lower-degree (higher-moment or ordinary) global symmetry: Recall the general degree- $(\mathrm{m}-1)$ polyno- mial global symmetry of Eq. (2.1),

$$
\Phi \rightarrow e^{i Q(x)} \Phi,
$$

where $Q(x)$ is a polynomial with degree at most ( $\mathrm{m}-1)$, say, Eq. (2.2),

$Q(x):=\left(\Lambda_{i_{1}, \ldots, i_{\mathrm{m}-1}} x_{i_{1}} \ldots x_{i_{\mathrm{m}-1}}+\cdots+\Lambda_{i, j} x_{i} x_{j}+\Lambda_{i} x_{i}+\Lambda_{0}\right)$.

We can reduce the degree-( $\mathrm{m}-1)$ higher-moment symmetry in $\mathbb{R}^{n}$ to a lower degree- $(\mathrm{m}-2)$ higher-moment symmetry in $\mathbb{R}^{n-1}$ by restricting to a specific subspace

$$
x_{i}=c_{i} .
$$

More generally, we can solve the polynomial with certain constraints as a lower-degree polynomial. This is related to the concepts of variety and subvariety in Sec. V A, and the mathematical concepts of embedding and foliation of subspaces. Indeed, the foliation concepts are powerful and applied recently in fracton literature; see, e.g., Refs. [46,47]. It is also pointed out that the concept of spacetime embedding may be treated as a quantum mechanical way as a quantized excitation, named the embeddon [4]. Therefore, it will be illuminating to revisit all the above gauge theories of highermoment or subdimensional polynomial global symmetry in a fully quantum mechanical setup in the future.

Note added: Companion pieces to this work include Ref. [4] and Ref. [36]. We thank Meng Cheng for pointing out potentially related work [34] and references therein on the study of the polynomial shift symmetries. This is a generalization to allow for an extension of the constant shift symmetry to a polynomial shift symmetry in the spatial coordinates. Although the essences of our ideas and theirs are related, the outcomes and motivations are dramatically different. We do not yet know the precise correspondence between our results and theirs.

\section{ACKNOWLEDGMENTS}

J.W. thanks Meng Cheng and Trithep Devakul for helpful communications on references in the literature. J.W. is supported by The Center for Mathematical Sciences and Applications at Harvard University. K.X. is supported by the Harvard Math Graduate Program and the Templeton Foundation's "The Black Hole Initiative: Towards a Center for Interdisciplinary Research." This work is also supported by NSF Grant DMS-1607871 "Analysis, Geometry and Mathematical Physics," and the Center for Mathematical Sciences and Applications at Harvard University.
[1] R. M. Nandkishore and M. Hermele, Fractons, Ann. Rev. Condensed Matter Phys. 10, 295 (2019).

[2] M. Pretko, X. Chen, and Y. You, Fracton phases of matter, Int. J. Mod. Phys. A 35, 2030003 (2020).

[3] H. Weyl, Elektron und Gravitation. 1, Z. Phys. 56, 330 (1929).

[4] J. Wang and K. Xu, Higher-rank tensor field theory of nonAbelian fracton and embeddon, Ann. Phys. 424, 168370 (2021).

[5] X.-G. Wen, Colloquium: Zoo of quantum-topological phases of matter, Rev. Mod. Phys. 89, 041004 (2017).
[6] C. Chamon, Quantum Glassiness in Strongly Correlated Clean Systems: An Example of Topological Overprotection, Phys. Rev. Lett. 94, 040402 (2005).

[7] J. Haah, Local stabilizer codes in three dimensions without string logical operators, Phys. Rev. A 83, 042330 (2011).

[8] S. Vijay, J. Haah, and L. Fu, A new kind of topological quantum order: A dimensional hierarchy of quasiparticles built from stationary excitations, Phys. Rev. B 92, 235136 (2015). 
[9] S. Vijay, J. Haah, and L. Fu, Fracton topological order, generalized lattice gauge theory and duality, Phys. Rev. B 94, 235157 (2016).

[10] D. J. Williamson, Fractal symmetries: Ungauging the cubic code, Phys. Rev. B 94, 155128 (2016).

[11] W. Shirley, K. Slagle, and X. Chen, Foliated fracton order from gauging subsystem symmetries, SciPost Phys. 6, 041 (2019).

[12] G. Savvidy and F. Wegner, Geometrical string and spin systems, Nucl. Phys. B 413, 605 (1994).

[13] G. K. Savvidy and K. G. Savvidy, Self-avoiding surfaces and spin systems, Phys. Lett. B 324, 72 (1994).

[14] R. Dijkgraaf and E. Witten, Topological gauge theories and group cohomology, Commun. Math. Phys. 129, 393 (1990).

[15] M. Kalb and P. Ramond, Classical direct interstring action, Phys. Rev. D 9, 2273 (1974).

[16] J. Wang, X.-G. Wen, and S.-T. Yau, Quantum statistics and spacetime surgery, Phys. Lett. B 807, 135516 (2020).

[17] P. Putrov, J. Wang, and S.-T. Yau, Braiding statistics and link invariants of bosonic/fermionic topological quantum matter in 2+1 and 3+1 dimensions, Ann. Phys. 384, 254 (2017).

[18] J. Wang, K. Ohmori, P. Putrov, Y. Zheng, Z. Wan, M. Guo et al., Tunneling topological vacua via extended operators: (Spin-)TQFT spectra and boundary deconfinement in various dimensions, PTEP 2018, 053A01 (2018).

[19] J. Wang, X.-G. Wen, and S.-T. Yau, Quantum statistics and spacetime topology: Quantum surgery formulas, Ann. Phys. 409, 167904 (2019).

[20] A. Kapustin and R. Thorngren, Anomalies of discrete symmetries in various dimensions and group cohomology, arXiv:1404.3230.

[21] J. C. Wang, Z.-C. Gu, and X.-G. Wen, Field Theory Representation of Gauge-Gravity Symmetry-Protected Topological Invariants, Group Cohomology and Beyond, Phys. Rev. Lett. 114, 031601 (2015).

[22] A. Rasmussen, Y.-Z. You, and C. Xu, Stable gapless Bose liquid phases without any symmetry, arXiv:1601.08235.

[23] M. Pretko, Subdimensional particle structure of higher rank U(1) spin liquids, Phys. Rev. B 95, 115139 (2017).

[24] M. Pretko, Generalized electromagnetism of subdimensional particles: A spin liquid story, Phys. Rev. B 96, 035119 (2017).

[25] M. Pretko, Higher-spin Witten effect and two-dimensional fracton phases, Phys. Rev. B 96, 125151 (2017).

[26] K. Slagle, A. Prem, and M. Pretko, Symmetric tensor gauge theories on curved spaces, Ann. Phys. 410, 167910 (2019).

[27] M. Pretko, The fracton gauge principle, Phys. Rev. B 98, 115134 (2018).

[28] S. Vijay and L. Fu, A Generalization of non-Abelian anyons in three dimensions, arXiv:1706.07070.
[29] H. Song, A. Prem, S.-J. Huang, and M. A. Martin-Delgado, Twisted fracton models in three dimensions, Phys. Rev. B 99, 155118 (2019).

[30] A. Prem, S.-J. Huang, H. Song, and M. Hermele, Cage-Net Fracton Models, Phys. Rev. X 9, 021010 (2019).

[31] D. Bulmash and M. Barkeshli, Gauging fractons: Immobile non-Abelian quasiparticles, fractals, and position-dependent degeneracies, Phys. Rev. B 100, 155146 (2019).

[32] A. Prem and D. J. Williamson, Gauging permutation symmetries as a route to non-Abelian fractons, SciPost Phys. 7, 068 (2019).

[33] J. Wang et al., Higher-rank tensor non-Abelian field theory: Higgs mechanism and beyond, arXiv:191n.nnnnn.

[34] T. Griffin, K. T. Grosvenor, P. Horava, and Z. Yan, Scalar field theories with polynomial shift symmetries, Commun. Math. Phys. 340, 985 (2015).

[35] A. Gromov, Towards Classification of Fracton Phases: The Multipole Algebra, Phys. Rev. X 9, 031035 (2019).

[36] J. Wang and S.-T. Yau, Non-Abelian gauged fracton matter field theory: Sigma models, superfluids, and vortices, Phys. Rev. Research 2, 043219 (2020).

[37] J.-K. Yuan, S. Chen, and P. Ye, Fractonic superfluids, topological vortices, and quantum fluctuations, Phys. Rev. Research 2 , 203267 (2020).

[38] N. Seiberg, Field theories with a vector global symmetry, SciPost Phys. 8, 050 (2020).

[39] D. Radicevic, Systematic constructions of fracton theories, arXiv: 1910.06336

[40] C. D. Batista and Z. Nussinov, Generalized Elitzur's theorem and dimensional reduction, Phys. Rev. B 72, 045137 (2005).

[41] Z. Nussinov and G. Ortiz, A symmetry principle for topological quantum order, Ann. Phys. 324, 977 (2009).

[42] Y. You, T. Devakul, F. J. Burnell, and S. L. Sondhi, Subsystem symmetry protected topological order, Phys. Rev. B 98, 035112 (2018).

[43] T. Devakul, D. J. Williamson, and Y. You, Classification of subsystem symmetry-protected topological phases, Phys. Rev. B 98, 235121 (2018).

[44] Y. You, T. Devakul, F. J. Burnell, and S. L. Sondhi, Symmetric fracton matter: Twisted and enriched, Ann. Phys. 416, 168140 (2020).

[45] T. Devakul, W. Shirley, and J. Wang, Strong planar subsystem symmetry-protected topological phases and their dual fracton orders, Phys. Rev. Research 2, 012059(R) (2020).

[46] W. Shirley, K. Slagle, Z. Wang, and X. Chen, Fracton Models on General Three-Dimensional Manifolds, Phys. Rev. X 8 , 031051 (2018).

[47] W. Shirley, K. Slagle, and X. Chen, Twisted foliated fracton phases, Phys. Rev. B 102, 115103 (2020). 